\title{
Rehabilitaciones urbanas en la ciudad contemporánea: entre las formas de hacer la ciudad y las formas de hacer en la ciudad ${ }^{*}$
}

\author{
Alicia Norma González de Castells \\ Universidade Federal de Santa Catarina (Brasil) \\ aliciacastells@superig.com.br
}

Resumen: Este artículo aborda, desde un punto de vista teórico, una reflexión en torno a la rehabilitación de centros históricos, barrios o calles en la ciudad contemporánea. Entre otros aspectos se analiza el efecto de homogeneización presente en las clásicas rehabilitaciones del tejido urbano de nuestras ciudades, tomamos también en consideración las diferentes dimensiones del espacio urbano, tanto simbólicas como de representación y, por último, presentamos las nuevas formas de apropiación de la ciudad contemporánea interrogándonos sobre el lugar que ocupa el patrimonio y el papel que este juega en la transformación de las intervenciones urbanas en atractivos para el consumo cultural y la inversión económica.

Palabras clave: Patrimonio, revitalización urbana, políticas públicas.

Abstract: This article studies, from a theoretical perspective, the regeneration of historical centres, neighbourboods and streets in contemporary cities. Among other aspects it analyzes the homogenizing effects of classic regenerations of the urban fabric of our cities and considers the different symbolic and representational dimensions of the urban space. It also presents new ways in which the contemporary city is being appropriated and asks questions about the place of heritage and the role it plays in making the urban environment an attractive space for cultural consumption and economic investment.

Keywords: heritage, urban regeneration, public policies.

\footnotetext{
* Una primera versión en portugués fue publicada en Alicia N. G. de Castells y Letícia Nardi (orgs.). Patrimônio cultural e cidade contemporânea. 1 ed. Florianópolis: UFSC, 2012, v. 1, p. 19-31.
} 


\section{Introducción}

Reflexionar sobre la rehabilitación de centros históricos, barrios o calles en la ciudad contemporánea exige asumir el presupuesto sobre la ciudad en cuanto construcción social. Significa concebir que las categorías que la ordenan y le dan sentido, como la de espacialidad, por ejemplo, sean sometidas a una continua mutabilidad. Trabajar, de hecho, con la idea de complejidad e incertidumbre de la ciudad sujeta a mudanzas sustantivas que acompañan su vida ciudadana y en gran medida sus paradigmas dominantes. Convivir con categorías provenientes de épocas consideradas de ruptura "[... ] tales como universal, modelo, homogeneidad, orden y previsión" que se contraponen a acciones de defensa de la ciudad existente y categorías excluidas del urbanismo moderno como calles, tejido urbano, espacio, lugar, etc., hoy objetos de investigación (Magalhães, 2007).

La tensión mencionada anteriormente puede ser ampliada sobre la visión del mundo contemporáneo defendida por Augé (1998). Concebido como unificado y plural, el adjetivo contemporáneo reflejaría el lugar donde coexisten tanto el número singular que este implica como la pluralidad de mundos que a su vez referencia (idem: 123). Para el autor, el nuevo orden de la realidad de mundos heterogéneos y relacionales encierra la misma paradoja: expresa tanto la singularidad que los constituye como la universalidad que los relativiza. Sobre esa trayectoria se corrobora la presencia y el reconocimiento de los niveles de complejidad e incertidumbre de que nos habla Magalhães (2007). Y nos permite entender todavía más la elección de la ciudad como un objeto privilegiado de estudio a fin de aprehender esta paradoja de nuevos mundos (Augé, ibidem: 125).

En cuanto a los intereses de esta reflexión, nos preocupa el efecto de bomogeneización presente en las rehabilitaciones del tejido urbano de nuestras ciudades, traducido en gran medida en la uniformización de las referencias culturales, de la mundialización de la información y de la imagen, que modifican "la naturaleza de la relación que cada uno de nosotros puede mantener con su entorno próximo y con su medio". Asumir, de hecho, la disolución de la idea de lugar que tanto preocupa a Augé (1994, 1998).

En ese escenario, entendemos que es esclarecedor pensar en el tipo de transmutaciones que pueden generarse, por ejemplo, en la rehabilitación de sitios históricos. Estos últimos, identificados como lugares de "vestigios" que guardan 
"las marcas del proceso de su construcción, de las transformaciones, adaptaciones, apropiaciones sufridas a lo largo del tiempo" y "de las relaciones que allí se sucedieron" (Motta, 2000: 260). Cuestionar —independientemente o no de tener sello patrimonial - lo que guardan de vestigios esos sitios históricos a ser preservados cuando son objeto de intervenciones continuas. Y aún más: cuáles son las posibilidades de resonancia (Gonçalves, 2005) de esos mismos sitios ante sus usuarios tradicionales.

Hablar de sitios, hablar de lugares, nos remite, a su vez, a la categoría de espacio, ampliamente discutida en la teoría social. Para Bourdieu (1979/1990), la existencia de formas de ordenación simbólica de las categorías de espacio y tiempo proporciona la estructura para las experiencias mediante las cuales aprehendemos quién o qué somos en la sociedad imponiendo al mismo tiempo en los individuos esquemas duraderos de percepción, de pensamiento y de acción. O como sostiene Urry (1991), las personas son altamente ligadas a diferentes espacios y resisten inclusive a la amenaza de su destrucción. Pero en ese nuevo orden de mundos heterogéneos soportados en altos niveles de complejidad e incertidumbre, ¿estaríamos (des)aprehendiendo quiénes somos o éramos? ¿Estaríamos sujetos a una disolución de nuestros esquemas de percepción pasados adoptando, tal vez, formas sustitutas y transitorias de percepción, de pensamiento y de acción que merecen ser objeto de análisis?

Retomando el foco de las nuevas configuraciones urbanas, Gupta y Ferguson (2000) defienden que, en un mundo globalmente interconectado, uno de los desafíos para los investigadores es encarar el enfoque sobre el modo como el espacio es imaginado (ipero no imaginario!). O sea, introducir la idea de lógicas espaciales en tensión dadas por el encuentro de los lugares imaginados a la distancia con sus transformaciones en cuanto espacios vividos. Levy y Segaud (1983) esclarecen todavía más la idea relativa a estas lógicas en tensión. Las autoras, sobre la base del presupuesto de que el ambiente no es fruto de un consumismo pasivo (en la línea argumentativa de De Certau, 1994), proponen un análisis de deconstrucción de ese mismo espacio en dos dimensiones que se corresponden con dos racionalidades - la simbólica y la de representación-. 


\section{Las racionalidades del espacio: simbólica y de representación}

La primera dimensión del espacio sería la que permite la inscripción del vínculo social a través de su representación, reflejada en la presencia de espacios "cualificados" por los propios actores, creando identidad entre el grupo social y el espacio. La segunda representa el espacio de Occidente, calificado como "representación". El espacio de representación sería un sistema que reposa sobre normas, axiomas y operaciones sacados de los fundamentos de la geometría euclidiana. Como espacio de representación, no posee ninguna diversidad de contenido, teniendo su homogeneidad dada por la identidad de la propia estructura. En contraste, dentro del primer espacio - lo figurativo- no se llega a aprehender la noción de espacio, pero a través de los procedimientos practicados por los propios practicantes, según Levy y Segaud, el espacio de representación se transforma, entonces, en un tipo de esquema mental en la conciencia occidental, un referencial universal para toda operación sobre el espacio. Y sus consecuencias se manifiestan en la impresión a través de correspondencias y homogeneizaciones que, por medio de mapas y planos de ordenamiento, transforman el territorio en una cuadrícula donde cada uno de sus puntos equivalentes no puede ser traducido dentro del espacio real. Esas dos nociones de prácticas dentro del espacio y el espacio como práctica de representación son también referenciadas por De Certeau (1994) cuando analiza las diversas modalidades que configuran las prácticas de los usuarios en lo cotidiano.

Vemos, entonces, que las rehabilitaciones urbanas que nos preocupan se encuentran en un estado de equilibrio inestable entre el espacio de "representación" concebido por los planificadores y las fuerzas hegemónicas y el espacio "cualificado" por el hombre común de que nos habla De Certau. La siguiente discusión ilustra esta afirmación.

\section{Nuevas formas de apropiación de la ciudad}

Al discutir el fenómeno denominado gentrificación, Zukin (2000: 98) sostiene que el proceso comenzó en los barrios urbanos históricos decadentes. Inicialmente, a través de un proceso de descubrimiento, por usuarios no residentes, de su "arcaísmo y belleza o autenticidades y diseño", consideradas por estos actores 
como formas de (des)valorizarlas porque estaban inscritas en construcciones contemporáneas propias de la población de estratos bajos, resultando en áreas urbanas progresivamente ocupadas y frecuentadas por nuevos consumidores. Es el caso, por ejemplo, de los artistas del Soho, en Nueva York, consumidores que son tanto productores de nuevos bienes culturales para consumo propio como de nuevas categorías culturales (idem: 88).

El proceso de gentrificación (o elitización) generalizado en áreas degradadas en nuestras ciudades es una práctica conocida por las acciones desarrolladas por las políticas públicas en complicidad —o con fronteras poco nítidas - con las fuerzas del capital privado (Smith, 2007; Bidou-Zachariasen, 2007; Proença, 2002). Las revitalizaciones pueden ser hechas tanto en centros históricos, calles o barrios tradicionales como en la realización de mejoramientos en áreas periféricas, cumpliendo a veces el papel de "higienizador" de dichas áreas. En todo caso, las revitalizaciones como estrategias políticas nunca son restringidas a ningún tipo de área y sí a los intereses políticos, económicos y comunitarios, entre algunas de las fuerzas más representativas de este fenómeno.

El proceso de apropiación cultural en cuanto estrategia de aumento del valor económico en las áreas centrales (Zukin, Maricato, Otilia Arantes, Vainer, Motta, entre otros) desencadena nuevas exclusiones y mayor control social. Otilia Arantes, refiriéndose al significado del término gentrificación, afirma que sus eufemismos "revitalización, rehabilitación, revalorización, reciclaje, promoción, recualificación, y hasta el mismo 'renacimiento', en realidad encubren la retomada del centro de las ciudades por las capas acaudaladas de las ciudades (Arantes, 2000: 31), redefiniendo el significado social de un lugar específicamente histórico para un segmento del mercado (Zukin, 2000: 87). Como también sugiere Delgado, transformando el espacio urbano en un campo temático "después de la debida expulsión de la historia" (Delgado, 2007:34).

Las intervenciones urbanas en Brasil pueden ser pensadas a partir de diferentes estrategias de acción y orientadas también por intereses divergentes (Maricato, 2001: 125). Valiéndose a propósito de la oposición de dos conceptos referentes a la intervención (el de renovación y el de rehabilitación) para distinguir y reforzar mejor sus diferencias, Maricato reconoce que, dependiendo del tenor de cada una de ellas, las acciones pueden ser inclusive mixtas. Asimismo, subraya que las acciones orientadas a la renovación son aquellas intervenciones hechas en la malla urbana que implican alta sustitución y construcción de nue- 
vas edificaciones marcadas por una estética posmodernista, y mayor ocupación y valorización del suelo urbano, lo cual provoca la expulsión tanto del pequeño comercio de características tradicionales como de la población residente. En el caso de la rehabilitación (o cualificación), las acciones procuran realizar intervenciones mínimas en la malla urbana para preservar el ambiente existente, como la permanencia de la población local. Observa que en los dos tipos de acciones citadas el patrimonio histórico, artístico y paisajístico considerado de "primera categoría" es preservado, mientras que en el segundo tipo de intervención lo preservado es el patrimonio común (idem, ibidem: 126).

Estas intervenciones - sujetas a preferencias y decisiones políticas que imprimen a su vez direccionamiento en la concepción, el gerenciamiento y la construcción de las obras-, los resultados arquitectónicos y urbanísticos y el tipo de apropiación social prevista no son tampoco, para Maricato, necesariamente idénticos. Intencionalmente, las experiencias alternan entre renovaciones asumidas como intervenciones "radicales" de esos procesos (gentrification) o rehabilitaciones que constituyen propuestas centrales de políticas nacionales (idem, ibidem: 142).

La dinámica de preservación del patrimonio cultural, la selección de lo que debe ser preservado, como veremos a continuación, sigue un rumbo que guarda semejanza con el cuadro explicitado por Maricato respecto de las intervenciones urbanas, pensadas en cuanto idealización y ejecución del todo.

\section{El lugar del patrimonio en las nuevas intervencio- nes urbanas}

En el contexto descrito, hay consenso entre los autores que discuten la problemática del patrimonio urbano (Jeudi, Arantes, Prats, Motta) de que las intervenciones urbanas actuales están transformándose en atractivos para el consumo cultural y la inversión económica. Antonio Arantes sostiene que lugares, paisajes y modos de vida son materia prima de un "sentido global de lugar" y que la conservación patrimonial, lejos de ser una acción neutra, es orientada técnica y políticamente a la dinámica cultural. Las iniciativas de preservación atienden a motivaciones específicas y se reflejan de modo también específico en relación con la población en general (Arantes, 2002: 84-85). 
También para Prats (2005), las activaciones patrimoniales han adquirido otra dimensión, entrando abiertamente en el mercado y pasando a ser valoradas por el consumo, "[...] medidor tanto de la eficiencia política como de la contribución al desarrollo o consolidación del mercado lúdico-turístico-cultural” (idem: 22). En el mundo contemporáneo, pautado por la arremetida de los medios de comunicación, de la publicidad y de la importancia de la imagen, dice Prats, el patrimonio participa también como un bien para el consumo visual (idem: 22-23), con lo cual Arantes resalta que muchas de las empresas y políticas de patrimonio dirigidas potencialmente para el mercado de esos bienes terminan por dar poca atención a la dimensión simbólica e invierten fuertemente en su aspecto alegórico. Según el autor, con el objetivo "de producir lugares para el mercado, ellas frecuentemente ponen en escena identidades de vitrina, para visitantes saturados de información, en escenarios desechables" (idem: 191).

Esta preponderancia del consumo visual es ilustrada por Vieira (2006). La autora discute los procesos de revitalización de áreas históricas desde la importancia de la imagen y del desarrollo de la industria turística a partir de tres estudios de caso - Pelourinho (Salvador), el barrio de Recife (Recife) y la Playa Grande (San Luis) - . Vieira señala que el proyecto de ciudad emprendido por los gobiernos locales se detiene cada vez más en la construcción de una imagen urbana positiva y atractiva a inversiones externas. Afirma que las intervenciones en los centros históricos por ella estudiados pertenecen a un tipo de "gentrificación inducida" (citando a Arantes), entendida como "procesos de elitización de áreas históricas a partir de capitales del Estado, y a veces en conjunto con la iniciativa privada". Para Vieira, las intervenciones no guardan un patrón ni un modelo de gestión único. Lo que se ha observado "son prácticas nocivas a la autenticidad del patrimonio edificado de estas áreas".

Motta (2000) hace un recorrido sobre la idea de preservación y tratamiento de los sitios históricos desde la óptica de su valor documental. Destaca que, contrariamente al modelo globalizado, esta perspectiva permite concebirlos como bienes patrimoniales "transformándolos en fuentes de conocimiento, referencias de la historia, de la memoria y de las identidades, elementos fundamentales al ejercicio de la ciudadanía" (2000: 257). De acuerdo con Motta, las prácticas de intervención y de proyectos que vienen siendo realizadas en áreas históricas de ciudades que disputan entre ellas el mercado globalizado orientado por estrategias de apropiación de estas para el consumo visual des- 
consideran "las ciudades como objeto socialmente construido y su patrimonio como fuente de conocimiento" (idem; ibidem: 257). La autora referencia los grandes proyectos impulsados por el Gobierno federal y los gobiernos estatales y municipales en Brasil: el Programa Monumenta/BID, promovido por el Ministerio de Cultura (MinC); la intervención del Proyecto Pelourinho, del gobierno estatal de Bahía, en Salvador; la propuesta de reurbanización de la plaza 15 de Noviembre, en Río de Janeiro, promovida por el gobierno municipal; y el Proyecto Colores de la Ciudad, de iniciativa privada, en el que participan la Fundación Roberto Marinho y fabricantes de pinturas. Concluye que muchas de esas intervenciones fueron escenario de tensiones entre sus patrocinadores y los órganos de preservación, inclusive aunque formaban parte de una misma instancia gubernamental. Motta aprovecha la oportunidad para delimitar varios de los problemas que este mencionado accionar provoca y que consideramos de la mayor gravedad: lo que estaría en juego serían los criterios a adoptarse en relación con las atribuciones de valor a los bienes escogidos; las formas de tratamiento de esos bienes; y el papel del poder público respecto a esas prácticas. Citando una de sus críticas:

[...] la intervención del poder público no puede caer en la dinámica del ofrecimiento del patrimonio cultural como mercancía, igualando el bien colectivo a los productos de consumo (idem; ibidem: 258).

\section{La dinámica del patrimonio social}

El estudio del fenómeno de la globalización en los países latinoamericanos (Rotman, 2004) muestra que, junto a los procesos homogeneizadores que tienen lugar en el mundo contemporáneo, ocurren también procesos diferenciadores en los cuales los nacionalismos y regionalismos adquieren relevancia y toman formas específicas de expresión. En ese contexto, la dinámica de lo global y de lo local se ha revitalizado; el tratamiento de tales categorías asumió una configuración que las posiciona de la condición de opuestas a una articulación más compleja (Rotman, 2004: 135).

Entendemos que esa articulación más compleja de la relación global-local, señalada por Rotman, cuando el foco del análisis es el patrimonio urbano en ciudades latinoamericanas, puede ser ilustrada por el estudio de Mantecón sobre el centro de la Ciudad de México. La autora, aun reconociendo que ha 
habido un crecimiento significativo de la periferia urbana a nivel demográfico, territorial y económico, creándose subcentros periféricos y desplazamientos de funciones y superposición de las funciones del centro para otras áreas, cree que existe un área central principal que acoge, además de seis siglos de historia urbana, una intensa combinación de usos económicos, políticos y culturales donde múltiples actores compiten por la definición de su vocación espacial. En el marco de esas especificidades locales, el proceso de transformación del área central, según Mantecón, supone la existencia de un conjunto de actores con diferentes estrategias interconectadas que no pueden ser interpretadas como resultado solamente de intereses económicos independientes y absolutos. Exige el reconocimiento de diferentes escenarios como son, por ejemplo, el accionar de las políticas públicas, la relación de los habitantes y de otros sectores de la sociedad civil con el patrimonio, y la presencia de capitales y sus alianzas por la conquista de nuevos espacios y esferas de acción en el área, donde el propio Estado puede ser visto como un interlocutor directo, ejerciendo un papel más decisivo en la cuestión patrimonial (Mantecón, <www.naya.org.ar>, acceso 26/6/07). La preocupación de Mantecón se refiere concretamente a la concepción que se tiene de la gestión del patrimonio. La relación plausible entre las formas de hacer la ciudad y las formas de hacer en la ciudad.

En esta dirección, Prats (2005) discute los procesos de construcción y de representación patrimonial como el de la especificidad de la categoría de patrimonio local, enfocando las características y las consecuencias de las intervenciones en procesos de activación y gestión del patrimonio local. Para él, desde la perspectiva de la construcción social del patrimonio, el patrimonio local, compuesto por todos aquellos objetos, lugares y manifestaciones locales, no debe presentar diferencias en relación con otros ámbitos de construcción patrimonial. Aunque el factor escala introduzca variaciones significativas tanto en su conceptualización como en la gestión (Prats, 2005; 23-24), el ámbito local le otorga al significado un carácter constituyente porque este se nutre "de la memoria, especialmente intersubjetiva compartida, construida a su vez a la luz de las diversas necesidades e intereses del presente" (idem; ibidem: 17). Pero, a la hora de la atribución de valor al patrimonio local, recuerda que las políticas de conservación y difusión del patrimonio lo conciben como una realidad esencial preexistente, no como una construcción social. Por lo tanto, de acuerdo con el mismo autor, la atribución de significado por parte de la población local a los 
bienes referenciales del patrimonio no corresponde a la atribución de valor y activación por parte de los poderes locales. Existen diferentes y contradictorios tiempos entre "la lógica del mercado a corto plazo y las necesidades, reales o percibidas como tales, que no necesariamente deben ajustarse a los tiempos políticos" (idem; ibidem: 27).

Cabe destacar que la categoría patrimonial (Prats, 1997), por tratarse de una construcción social, no puede ser pensada como algo dado ni considerada como un fenómeno universal. Proença (2002), en un estudio sobre la "revitalización” urbana del Recife Antiguo, argumenta que, en esos tipos de intervención que presuponen segregación social y vaciamiento de los espacios públicos, "los usos y contra-usos que en ellos se estructuran compiten inversamente para su reactivación como espacios públicos" (2002: 116). Desde esta perspectiva, el patrimonio cultural debe ser concebido como un artificio, ideado individual y colectivamente, con la condición de que puede ser históricamente mutable, con nuevos intereses que determinen nuevos fines y nuevas circunstancias (Prats, 1997: 20). Y que, así, no dependa apenas de voluntades individuales o de grupos para encontrar resonancia junto a su público (Gonçalves, 2005).

\section{Consideraciones finales}

Para finalizar, entendemos que los procesos de patrimonialización desencadenados en las nuevas configuraciones urbanas, sujetos a la uniformización y al alto consumo visual, no deben ser interpretados como resultado solamente de intereses económicos independientes y absolutos. Una relación puramente dicotómica entre el patrimonio como referencia o como recurso. Las rehabilitaciones urbanas que nos preocupan se encuentran en un estado de equilibrio inestable entre el espacio de "representación" concebido por los planificadores y las fuerzas hegemónicas y el espacio "cualificado" por el hombre común del que nos habla De Certeau. Se puede afirmar, así, que en las formas de hacer la ciudad los bienes patrimoniales también participan (Prats, Rotman, Canclini, Mantecón, Arantes, entre otros). Y que para dar cuenta de las especificidades locales de esos procesos de transformación es preciso el reconocimiento de los diferentes "escenarios" que componen el campo del patrimonio.

Solamente así creemos que es posible lanzar una luz fecunda sobre las paradojas contemporáneas a las que aludíamos en el inicio de este trabajo. Fi- 
nalmente, parafraseando a Augé, la ciudad es, sin duda, tanto escenario de la representación de la singularidad que la constituye como de la universalidad de la relativiza.

\section{Referencias}

Arantes, Antônio (org.). O espaço da diferença. Campinas, São Paulo: Papirus, 2000 .

- "Cultura, ciudadanía y patrimonio en América Latina”. En: Lacarrieu, Mónica y Álvarez, Marcelo (comps). La (indio)gestión cultural: una cartografía de los procesos culturales contemporáneos. Buenos Aires: Ed. Ciccus/ Ed. La Crujía, 2002.

Arantes, Otília. "Uma estratégia fatal: a cultura nas novas gestões urbanas". En: Arantes, O; Vainer, C. y Maricato, E. (org.). A cidade do pensamento único: desmanchando consensos. Petrópolis, Río de Janeiro: Vozes, 2000.

Augè, Marc. Não Lugares. Introdução a uma antropologia da supermodernidade. Campinas, São Paulo: Papirus, 1994.

- Hacia una antropología de los mundos contemporáneos. Barcelona: Gedisa, 1998.

Bidou-Zachariasen, Catherine. De volta à cidade. São Paulo: Annablume, 2007.

Bourdieu, Pierre. Coisas Ditas. São Paulo: Brasiliense, 1990.

- La distinción. París: Minuit, 1979.

Canclini, Néstor García. Culturas híbridas. Estratégias para entrar e sair da modernidade. Nueva Edición. Buenos Aires: Paidós, 2005.

Castells, Alicia N. G. de. "Vida cotidiana sob a lente do pesquisador: o valor heurístico da imagen”. Antropologia em Primeira Mão. UFSC, diciembre de 1999.

- "O estudo do espaço na perspectiva interdisciplinar". Antropologia em Primeira Mão, v. 47, p. 1-20, 2001.

- "A criatividade dos Sem-Terra na construção do habitat. Um olhar etnográfico sobre a dimensão espacial do MST". Tesis de Doctorado - Doctorado Interdisciplinar en Ciencias Humanas - Universidade Federal de Santa Catarina - UFSC, Florianópolis, 2001. 
Certau, M. de. A invenção do cotidiano. Artes de fazer. Río de Janeiro: Vozes, 1994.

Delgado, Manuel."Barcelona: o mito do espaço público”. En: Humboldt, apropriações da cidade. Revista semestral, año 49, núm. 95, Goethe-Institut, 2007.

Gonçalves, José Reginaldo dos S. "Ressonância, materialidade e subjetividade: as culturas como patrimonios". Revista horizontes Antropológicos. Porto Alegre, año 11, núm. 23, p. 15-36, enero/junio 2005.

Gupta, Akhil y Ferguson, James. "Mais além da cultura: espaço, identidade e política da diferença”. En: Antônio Arantes (org.). O espaço da diferença. Campinas, São Paulo: Papirus, 2000.

Jeudy, Henri-Pierre. Memórias do social. Río de Janeiro: Forense Universitária, 1990.

- Espelho das cidades. Río de Janeiro: Casa da Palavra, 2005.

Lévy, Françoise y Segaud, Marion. Antropologie de L'espace. París: Centre Georges Pompidou CCI, 1983.

Magalhães, Sérgio Ferraz. A cidade na incerteza: ruptura e contiguidade em urbanismo. Río de Janeiro: Viana \& Mosleyः Ed. Prourb, 2007.

Mantecón, Ana Rosas. Las jerarquías simbólicas del patrimonio: distinción social e identidad barrial en el Centro Histórico de la Ciudad de México. <http:// www.naya.org.ar>. Acceso 26/6/07.

Maricato, Ermínia. Brasil, cidades. Alternativas para a crise urbana. Petrópolis, Río de Janeiro: Vozes, 2001.

MotтA, Lia. "A apropriação do patrimônio urbano: do estético-estilístico nacional ao consumo global". En: Antônio Arantes (org.). O espaço da diferença. Campinas, São Paulo: Papirus, 2000.

Prats, Llorenç. "Concepto y gestión del patrimonio local”. En: Cultura y patrimonio, perspectivas contemporáneas en la investigación y la gestión. Cuadernos de Antropología Social. Sección de Antropología Social, núm. 21, Buenos Aires, julio de 2005.

Proença, Rogério Leite. "Contra-usos e espaço público: notas sobre a construção social dos lugares na Manguetown". En: RBCS, vol. 16, núm. 49, junho/2002.

Rotman, Mónica B. "Globalización y patrimonio cultural: la recreación de identidades locales". En: RUNA, Archivos para las ciencias del hombre/UBA. 
Facultad de Filosofía y Letras, Instituto de Ciencias Antropológicas, núm. 24, Buenos Aires, 2004.

Sмiтн, Neil. "A gentrificação generalizada: de una anomalia local à «regeneração» urbana como estratégia urbana global”. En: Bidou-Zachariasen, Catherine. De volta á cidade. Dos processos de gentrificação às políticas de "revitalização" dos centros urbanos. São Paulo: Annablume, 2007.

URrY, John. "Time and space in Giddens Social Theory”. En: Giddens' Theory of Structuration. Londres: Ed. Christopher G.A. Bryant and David Jary, 1991. Vainer, C. "Pátria, empresa e mercadoria. Notas sobre a estratégia discursiva do Planejamento Estratégico Urbano". En: Arantes, O.; Vainer, C. y Maricato, E. (orgs.). A cidade do pensamento único: desmanchando consensos. Petrópolis, Río de Janeiro: Vozes, 2000.

Vainer, C. y Maricato, E. (orgs.). A cidade do pensamento único: desmanchando consensos. Petrópolis, Río de Janeiro: Vozes, 2000.

Vieira, Natalia."Autenticidade x exploração imagética do patrimônio cultural. Trabalho apresentado no Grupo de Trabalho: Patrimônio e cultura: processos de politização, mercantilização e construção de identidades”. En: CD de la $25^{\circ}$ Reunião Brasileira de Antropologia, 11 a 14 de junio. Goiânia. Goiás. Brasil, 2006.

Zukin, Sharon. "Paisagens do século xxi: notas sobre a mudança social e o espaço urbano". En: Arantes, A. (org.). O espaço da diferença. Campinas, São Paulo: Papirus, 2000. 